\title{
Analogizing: The Literary Relevance of Holyoak and Thagard's Mental Leaps
}

Anders Pettersson 
In 1995, the psychologist Keith J. Holyoak and the philosopher Paul Thagard published a book about analogical thinking, its structure, and its uses entitled Mental Leaps: Analogy in Creative Thought. In my paper I will first introduce some of Holyoak and Thagard's main ideas and then go on to reflect on the possible usefulness of their theoretical perspective for the understanding of the art of literature.

In the call for papers, we were invited to consider, among other things, "contemporary aesthetics ... and its place among the humanities and in the larger culture". I see my paper as being related to the conference theme not least because of its foregrounding of the significance that cognitive poetics and empirical research can have for contemporary literary aesthetics.

Several different cognitive approaches - which are not necessarily mutually exclusive - are of patent interest for the explication of the reading and understanding of literature. Two recent books that immediately come to mind are Gilles Fauconnier and Mark Turner's The Way We Think: Conceptual Blending and the Mind's Hidden Complexities (2002), centred around the concept of blending, and George Currie and Ian Ravenscroft's Recreative Minds: Imagination in Philosophy and Psychology (2002), where the focus is on concept of simulation. When the subject is analogical thinking, the extensive work on metaphor by George Lakoff and his various associates is certainly very relevant, and considering the centre of attention that will emerge later, Mark Turner's discussion of literary works as parables in his book The Literary Mind (1996) is even more so. For reasons of space, however, I will have little to say about other cognitive theorists than the couple I have chosen to deal with on this occasion.

\section{II}

No formal definition of "analogy" is to be found in Mental Leaps. Holyoak and Thagard introduce analogical thinking by describing it as the attempt to understand a given phenomenon, the target analog, by perceiving it in terms of a more familiar phenomenon, the source analog (p. 2). Their opening example is a four-year-old boy, Neil, who ponders the question of what birds might use for chairs. I quote:

Neil suggested, reasonably enough it would seem, that a tree could be a bird's chair. A bird might sit on a tree branch. His mother said that was so and added that a bird could sit on its nest as well, which is also its house. 
The conversation went on to other topics. But several minutes later, the child had second thoughts about what a tree is to a bird: "The tree is not the bird's chair - it's the bird's backyard!" (ibid.)

Here, familiarity with human houses, and the idea of some kind of structural similarity between the dwellings of humans and birds are used to create a frame for the understanding of the housing of birds. Human houses and their surroundings function as the source analog; birds' nests and their surroundings form the target analog.

In Mental Leaps, Holyoak and Thagard describe fundamental conceptual mechanisms underlying analogizing, "the basic requirements for abstract thinking, the capacities that a mind must have to be able to break loose from sensory experience and create complex models of the world" (p. 19). Further, they define types of analogizing of varying structural complexity, types that they call attribute mapping, relational mapping, and system mapping (Chap. 2, esp. pp. 26-31). They explain to what extent apes have the capacity to analogize, and how children gradually acquire the skill (Chaps. 3-4). Basing themselves, in part, on empirical experiments, they show how analogies are utilized in explanation and understanding and in decision making (Chaps. 5-7). They also present "a collection of many of the most important analogies that scientists have used", and they offer "an account of the main mechanisms required for analogical thinking in science" (chap. 8, citation from p. 185). They attempt to point to typical constraints on analogizing, and they sketch computer simulations of analogical thinking understood as an instance of parallel constraint satisfaction (chap. 10, esp. pp. 240-256). In addition, they pay attention to the general role of analogy in culture and to its use in the arts, not least in literature (esp. chap. 9).

For Holyoak and Thagard, analogical thinking is a species of problem solving. The process of analogizing has a typical basic structure: "Often a problem solver will select a source analog by retrieving information about it from memory (selection), map the source to the target and thereby generate inferences about the target (mapping), evaluate and adapt these inferences to take account of unique aspects of the target (evaluation), and finally learn something more general from the success or failure of the analogy (learning)." (p. 15)

As I have already noted, Holyoak and Thagard view analogizing as a species of comprehending of unfamiliar matters in terms of familiar ones. According to them, analogizing pervades human 
thinking: "Comparing novel situations to familiar ones and finding correspondences between them, and then using these correspondences to generate inferences about the new cases, is integral to human thinking." (p. 262)

It is true, of course, that analogies in themselves prove nothing: "Analogy must be recognized as a source of plausible conjectures, not irrefutable conclusions. The success of an analogy must finally be judged by whether the conjectures it suggests about the target analog prove accurate and useful." (p. 7) "Analogies should enhance thinking, not substitute for it." (p. 133)

As I mentioned in passing, Holyoak and Thagard themselves have ideas about the roles that analogical thinking plays in literature. Not surprisingly, they note how analogy forms the basis of figurative language, not least of metaphor (pp. 213-227). But they also ascribe a more global role to analogizing: they suggest that literary works are typically used, among other things, as source analogs from which readers construct analogies targeting the real world. In analogical thinking generally, the analogizer faces a given phenomenon which appears difficult to understand, the target, and has to find a source analog able to suggest productive ways of perceiving the target. In connection with literature, the situation is, in a way, reversed: the reader "encounters a text that may provide a metaphorical source and often has to discover the target domain that is the underlying topic". (p. 225)

\section{III}

In my reflections on the literary relevance of Holyoak and Thagard's analysis of analogical thinking, I will concentrate on analogizing in the "global" role just referred to. It is helpful to have a literary example to refer to. Let us think of Nadine Gordimer's novel The Pickup (2001).

The Pickup is a story about a young South African woman living in Johannesburg, Julie Summers, the daughter of a wealthy and influential father on whom she has more or less turned her back. When her car happens to break down she meets an illegal immigrant from Yemen, Ibrahim ibn Musa, who works as a mechanic in the garage to which she turns for help. Little by little they get to know one another, and they become lovers. When Ibrahim is discovered by the authorities and eventually deported back to Yemen, Julie marries him, gives up her work, follows him back to his country, and attempts to start a new life in his family home. At the end of the novel, Ibrahim finally procures visa for the United 
States for himself and Julie, but she decides to stay in Yemen with his parents and siblings while he leaves, alone, for America.

The reviewers did not all focus on the same aspects of The Pickup. For example, Susie Linfield pointed especially to "the desperate power inequities between haves and have-nots created by the new globalism". The subject brought to the foreground by Andrew Sullivan was that of "two cultures in search of each other" whose "mutual incomprehension is also related to their mutual attraction". Magnus Eriksson, for his part, commented first and foremost on Gordimer's criticism of "a lifestyle that has made the children so rootless with respect to values that they may be attracted to an authoritarian structure in society and family". The formulations cited are the closest one gets, in the reviews by Linfield, Sullivan, and Eriksson, to descriptions of the theme of The Pickup, of the novel's "main point, message, or moral", as one recent definition of the concept of a theme puts it.

It is obvious from the respective contexts that the reviewers are referring to the real world with "power inequities", "cultures", "lifestyle", et cetera, not to the fictional world. They relate aspects of the fictional world to aspects of reality. Clearly, part of what the reviewers do is point to analogies, or supposed analogies, between the world of the novel and the real world.

A newspaper critic does not of course function as an ordinary reader pure and simple, but it can hardly be doubted that the kind of analogizing just illustrated is a common feature of literary reading. Different types of evidence, included that from empirical investigations, could be adduced in support of this generalization.

The themes of literary works are naturally important factors in literary transactions, so we seem to have discovered an aesthetically and artistically significant field of application for a theory of analogical thinking. It may be objected, though, that themes are integral elements of literary works in that they exist before the reader comes into the picture. Let us call this the "Part-ofutterance-meaning Theory of Themes". To develop that objection; identifying the theme of a novel like The Pickup is part of comprehending what the work means. Analogical thinking may play some not uninteresting part in that connection, but its role will certainly be rather narrowly circumscribed and ultimately subsidiary to the establishment of work meaning, however that is thought to be achieved.

The "Part-of-utterance-meaning Theory" represents one way of conceiving of a work's main point, message, or moral. Another 
possibility is to think of themes not as part of the work's meaning in the sense just introduced, but as part of the work's significance, part of the readers' ultimately subjective perception of the work's relevance and importance for them. Let us call this the "Part-of-significance Theory of Themes". To develop that alternative; identifying the theme of a literary work like The Pickup is a matter of reading and understanding the work (establishing the work meaning as one goes ahead) and simultaneously making connections between the work and real-world affairs of some relevance to oneself. Analogical thinking may be presumed to play a key role in the identification of themes.

The Part-of-utterance-meaning Theory of Themes is often preferred within analytical aesthetics. It is adopted, for example, by James O. Young in his recent book Art and Knowledge (2001). Young says - much like Holyoak and Thagard - that a work of art "gives us a way of looking at the world. In order to test the work, we need to look at the world in this way". The way of looking at the world that a work of art may provide is however, for Young, not something created by the reader in whole or in part. It is something the work represents, which means that it is an objective feature of the work merely recovered by the reader.

I can see two main problems - or one composite problem - with part-of-utterance-meaning theories of themes. First, they never seem to be able to demonstrate to my satisfaction how we are to arrive at the supposedly correct identification of a theme. They indicate a general mechanism - according to Young, for example, all works of art and literature represent by means of something which he calls "illustrative representation" - but without explaining in any detail the step or steps by which the mechanism leads us, in the individual case, from manifest textual meaning to implied theme. In other words, a theme-generating mechanism is hypothesized, but its workings are neither clearly explained nor concretely demonstrated. This tends to give part-of-utterancemeaning theories of themes a somewhat apodictic character.

This would not perhaps have been so serious if readers had been relatively unanimous in their identifications of themes, as they normally are in their identification of the manifest verbal meaning of texts. If that were the case it would be natural to suppose that implicit thematic meaning is indeed objective, although it emerges in ways which are not completely understood. In fact, however, different readers typically do not discern exactly the same theme in a literary work.

The three critics mentioned earlier provide an example of this: 
Linfield focused on "inequities between haves and have-nots", Sullivan on "cultures in search of each other", and Eriksson on "a lifestyle that has made the children ... rootless". I would expect part-of-utterance-meaning theories of themes to supply us with instruments for deciding who, if anyone, is right in a case like this. The looser the analysis is - the less it contributes to resolving such questions - the more it appears vacuous to me.

Objections to part-of-utterance-meaning theories of themes in this vein are not original. For example, Victoria Kurtz and Michael F. Schober reported, in 2001, a study of sixteen readers identifying themes under controlled conditions, and concluded, on the basis of the diversity of the identifications of themes arrived at by their subjects: "The results strongly suggest that themes do not reside in texts in any obvious way but are constructed by readers."

Advocates of a part-of-utterance-meaning theory of themes could certainly find a whole array of counter-arguments. For example, the aesthetic relevance of what I have here called significance could be disputed, Kurtz and Schober's study could be critically scrutinized, and the felicitousness of the examples from Linfield, Sullivan, and Eriksson questioned. I by no means claim to have disposed of the Part-of-utterance-meaning Theory of Themes, even if I personally would be more inclined to support the Part-of-significance Theory. I say "more inclined", since I do not actually believe that the Part-of-significance Theory can do justice to the complexity of the situation, either - not at least in the rather unsophisticated version that we have been considering. However this is not the place to pursue these issues.

\section{IV}

What, then, has come out of the discussion? I have pointed to examples of how identification of themes in a literary work must be supposed to be grounded in analogical thinking, and I have remarked that the aesthetic importance of that observation will be greater if we adhere to a part-of-significance theory of themes than if we subscribe to a part-of-utterance-meaning one. The two theories can certainly be refined. Arguably, the aesthetic importance of the phenomenon of analogizing will, on the whole, increase the more the analysis approaches the part-of-significance end of the spectrum.

Leaving the idea of hard proof to one side, let us assume for the sake of discussion that themes that are constructed and not 
just recovered by readers will have to be admitted a role, larger or smaller, in a satisfactory account of paradigmatic reading of literature. In what then would the literary relevance of Holyoak and Thagard's analysis of analogical thought consist?

If we are to work, to a greater or lesser extent, with a part-ofsignificance analysis of themes, we need to be able to explain how readers construct themes and kindred phenomena. If themes are supposed to constitute the "main point, message, or moral" of a literary work this should be a task key to the understanding of literature as an art.

Several mechanisms have been proposed for explaining what we could call "constructive thematization". The largest family of explanations can be brought together under the vague heading of "identification". Explicit explanations of this general type go back at least to Freud's lecture on creative writers and daydreaming, and this avenue of research is constantly in use and constantly in transformation.

Other mechanisms are more cognitive in character, such as Gadamer's theory of application or the theories of Nussbaum and others about literature as a source of ethical knowledge (normally thought to be tapped, it is true, through a kind of identification, a kind of imaginative participation in the fictive events by the reader). Walton's analysis of fiction processing in terms of games of make-believe, now seemingly eclipsed by the more distinctly identification-coloured idea of simulation, could also be mentioned in this connection.

Analogizing, in Holyoak and Thagard's sense, joins this fairly large group. The different models need not necessarily be competitors. It is, however, relevant to ask what analogizing might contribute to our understanding of the area, and where its main weaknesses may be suspected to lie.

In my view, Holyoak and Thagard's analysis of analogical thinking has two especially strong points. First, the analysis is based on mainstream cognitive psychology, and thus helps to build a bridge between aesthetic theory and empirical disciplines studying human behaviour; it is also supported to a considerable extent by concrete empirical evidence. In these respects it is similar to the idea of identification but distinguishes itself from, for example, Gadamer's idea of literary reading as application or the idea of fiction reading as a game of make-believe.

Second, analogical thinking is a very wide and versatile concept. It is wider than identification (whether identification is presented under the label of "identification", of "empathy", or of 
"simulation"). Identification could in fact be called a variety of analogical thinking. Identification is always a case of analogizing - a connection between the other and the self is established and explored - but the converse is not true: analogical thinking can be carried out without identification. (For example, to my mind at least, it would be to overextend the concept of identification to say that four-year-old Neil in our initial example identified with a bird when he speculated about what birds use for chairs.)

The wide and versatile nature of the concept of analogical thinking may be thought of as a dubious blessing: it is easy to feel that the concept is so inclusive that it has little explanatory power. I suspect that its value for an analysis of constructive thematization in the reading of literature will lie predominantly in its capacity to offer a productive frame able to hold together otherwise dissimilar activities and relate them to one another. The more one understood this kind of thematization to be a multifarious phenomenon, a bundle of related activities, the more important that quality would be.

Even when it is viewed in this perspective, Holyoak and Thagard's analysis of how readers may use literary works as source analogs appears to be very sketchy. The lack of development is entirely understandable, since the analysis occupies a rather marginal position in Holyoak and Thagard's book. Yet the analysis certainly leaves many interesting questions unasked about analogical thinking in the constructive thematization of literature.

Some of these are empirical. For instance: Is it actually true that readers analogize (as I took for granted earlier)? Supposing they do, what, more precisely, do they get out of it? Can readers' analogizing be said to be of different types?

Other questions are, at bottom, normative; for example: Is analogizing always aesthetically relevant, or always aesthetically irrelevant, or sometimes relevant and sometimes irrelevant (and how are we to decide whether or not a given instance of analogical thinking in connection with themes is aesthetically relevant)?

Finally, there is also the fascinating, partly empirical, partly evaluative question: Does literature, as it is encountered in ordinary reading for literary enjoyment, have special, more or less unique merits (and drawbacks?) as a source analog, and if so: what merits, and why?

I am convinced that philosophical aesthetics, empirical aesthetics, and cognitive science all have important contributions to offer when we attempt to answer such questions. 